\title{
EL CONCEPTO DE ENERGÍA EN LOS LIBROS DE TEXTOS: DE LAS CONCEPCIONES PREVIAS A LA PROPUESTA DE UN NUEVO SUBLENGUAJE
}

\author{
MICHINEL MACHADO, J.L. y D'ALESSANDRO MARTÍNEZ, A. \\ Escuela de Física, Facultad de Ciencias, UCV, 47586 Caracas 1041-A. \\ Cátedra de Fisiología, Escuela Luis Razetti, Facultad de Medicina, UCV. \\ Departamento de Procesos Biológicos, USB.
}

\begin{abstract}
SUMMARY
In this work are studied the already existing interpretations and conceptions on the concept of energy and other allied concepts in Basic Physics textbooks commonly used in the initial courses at university and in courses prior to university level (Basic School) being given in Venezuela.

In this study we have used techniques of analysis of content and have made a historic analysis to round up the conclusions of the work.

It becomes necessary to build a new language, simple but internally coherent, and within the current scientific paradigms. In this work we start the discussion of this new language within the area of energy and related concepts.
\end{abstract}

\section{INTRODUCCIÓN}

En los últimos tiempos se han realizado estudios de los factores que caracterizan el aprendizaje de las ciencias. Algunos de estos estudios (Sebastiá 1987, Moreira 1982, Erickson 1979) han puesto de manifiesto la existencia de interpretaciones previas o preconcepciones, por parte de los estudiantes, de los fenómenos físicos que pudieran afectar el aprendizaje de la física. Entendemos las preconcepciones (o conceptos precientíficos) como interpretaciones de los fenómenos físicos que son hechas por los individuos a partir del uso de «evidencias de sentido común» (Gil 1991) como proceso de razonamiento; entendiendo el «sentido común» como un sistema de razonamiento que consiste en: $a$ ) establecer conclusiones a partir de observaciones eminentemente cualitativas y no controladas, b) extrapolar sus uevidencias", aceptándolas acríticamente (Piaget 1969, Gil 1991), c) oposición al proceso científico de construir el conocimiento, en el entendimiento de que el sentido común está caracterizado por la certidumbre, por la ausencia de dudas, no toma en cuenta el pensamiento divergente o la existencia de soluciones alternativas, se centra en el tratamiento de situaciones puntuales sin hacer uso del razonamiento inductivo-deductivo y no procesa información que es obtenida a través de la experimentación controlada. Estas preconcepciones conducen al estu- diante, en la mayoría de los casos, a falsas concepciones de los fenómenos.

Por otro lado, parte de las evaluaciones de los componentes del sistema educativo han sido dirigidas, en nuestro país, hacia el libro de texto (Sebastiá 1982, Qüenza 1986) en sus aspectos de contenido (calidad científica, consistencia lógica y psicológica) y de pre. sentación (adecuación a la audiencia, congruencia curricular, aspectos tecnográficos y lingüísticos). También en otros paŕses se han realizado estudios que consideran el contenido de los textos (Lehrman 1982a, Lehrman 1982b).

En este trabajo, a la luz de la teoría cognitiva de D. Ausubel (Ausubel, Novak y Hanesian 1980), se diagnostica la presencia de preconcepciones de la física, y concretamente relativas a la energía. Distintas investigaciones han sido realizadas en el diagnóstico de concepciones previas, relativas al concepto de energía y a otros conceptos que le son afines, poseídas por los estudiantes, así como de propuestas didácticas que conduzcan al cambio conceptual de éstos (Erickson 1979, Duit 1984, Solomon 1983, Watts 1983, Drivery Warrington 1985). Pero, poco o nada ha sido hecho con el fin de 
constatar la presencia de las concepciones previas en los propios autores de libros de texto, transformándose en fuentes generadoras de concepciones previas de los estudiantes.

La importancia que las teorías cognitivas asignan al conocimiento previo del estudiante para el desarrollo de un aprendizaje significativo nos lleva a prestar atención a lo que se conceptualiza en los textos. Las interpretaciones de los fenómenos fúsicos que expresan los libros de texto tienen un doble efecto en el desarrollo del conocimicnto del estudiante: el que se produce por la lectura directa por parte đe éste y el que le presenta el profesor que también hace uso del texto.

Además, el lenguaje utilizado por la física o sublenguaje de la física (Touger 1983) tiene una influencia determinante sobre los autores de los textos, ya que ellos deben presentar la interpretación de los fenómenos científicos en base a determinados paradigmas, haciendo uso de un sublenguaje que le es propio. En este particular se constata la presencia de un sublenguaje característico de teorías físicas no vigentes (el mecanismo de la energía y la teoría del calórico) como medio de expresar los paradigmas vigentes, resultando una evidente contradicción.

A pesar de haber transcurrido casi dos siglos de los experimentos de Benjamín Thompson (conde de Rumford 1807), del establecimiento de la ley general de la conservación de la energía por Mayer, Helmholtz y Joule (1842-1847) y del desamollo de la teoría cinética de los gases, entre otros, por Clausius, Maxwell, Kronig y Boltzmann (en la segunda mitad del siglo pasado), gran cantidad de artículos científicos y libros de texto de diferentes niveles de la enseñanza aún están orientados con términos y concepciones provenientes de la teoría del calórico. La persistencia de estas ideas tiene diversas causas; una de ellas, la cual constituye una de nuestras hipótesis de trabajo, tiene su origen en la histórica polémica onda vs partícula, energía vs fuerza, interacción a distancia vs interacción partícula partículas, como modelos válidos para interpretar los fenómenos físicos y que, evidentemente, no está resuelta. Además, existen razones inherentes al concepto de energía relacionada con la polémica entre la metateoría idealista del energetismo (Boltzmann 1986) ( La energía y no la materia es la sustancia del mundo físicos) desarrollada por Ostwald, Mach y Duhem (1902) y los mecanicistas. Esta filosofía y la misma polémica han influido negativamente en la difusión de la formalización del concepto de energía y hasta en el propio desarrollo de dicho concepto.

En esta investigación documental en la que se analiza el discurso de una serie de autores, se utitizan técnicas de análisis de contenido, complementándose con un análisis historico del desarrollo del concepto de energía y otros que le son afines.

Se detectan interpretaciones que pueden ser clasificadas en tres tipos:

- Un primer grupo que expresa deficiencias del autor en el conocimiento de las teorías físicas vigentes.
- Un segundo grupo con características típicas de las preconcepciones.

- Un tercer grupo que refleja la existencia de un sublenguaje, ya tradicional, pero deficiente, donde influye en forma determinante, por un lado, la permanencia de la concepción mecanicista de la energía (por ejemplo, la energía es la capacidad para hacer trabajo) y, por el otro, la teoría del calórico. La persistencia de tal sublenguaje, creemos que se debe a que abrevia o simplifica, en algunos casos, la explicación de ciertos fenómenos físi. cos. Tal abreviación o simplificación, aunque puede tener utilidad didáctica, refuerza las preconcepciones.

Se hace necesario construir un nuevo ienguaje, sencillo, pero coherente internamente y con los paradigmas científicos vigentes. En este trabajo iniciamos la discusión de ese nuevo lenguaje en el area de la energía y conceptos afines.

\section{MATERIALES Y MÉTODOS}

\section{Técnica de análisis del contenido}

En vista de que esta investigación es una indagación documental en Ia que se analiza el discurso de una serie de autores, se utilizó la técnica de análisis de contenido (Ander-Egg 1980, Berelson 1952), que es un método de investigación para el estudio objetivo, sistemático y cuantitativo del contenido manifiesto en las comunicaciones, en la cual se efectúa una recopilación de datos que permite obtener información o tendencias contenidas en ellas, con el propósito de estudiar ideas, significados, temas o frases, y no las palabras o estilos con las que éstas se expresan. La descripción detallada de la técnica no es pertinente en este momento.

\section{Muestra bibliográfica}

En este trabajo se analizó una muestra (Tabla I) que consiste en cinco libros de física básica del nivel universitario y diecisiete libros de texto que son utilizados para las asignaturas Estudio de la Naturaleza y Física en cursos previos (escuela básica) a la universidad.

\section{Los conceptos de energía y calor}

Para poder hacer el análisis de los textos fue necesario establecer definiciones paradigmáticas de la energía y del calor. Trabajos de distintos autores (Lehrman 1973 , Lehrman 1982a, Lehrman 1982b, Zemansky 1970 , Barrow 1988, Holton 1986, Michinel, D'Alessandro y Ortega 1992, Michinel y D'Alessandro 1993) nos han permitido establecer estas definiciones, a partir de las características que le son propias, en los siguientes términos:

«Energía es una magnitud física que se presenta bajo diversas formas, está involucrada en todos los procesos 
Tabla I

Libros revisados

\section{Universitarios}

- Resnick, Robert y Halliday, David, Fisica (Parte 1), Editorial Continental, 1983.

- Sears, Francis y Zemansky, Mark, Física, Editorial Aguilar, 1971.

- Serway, Raymond, Física, Editorial Interamericana, 1985.

- Alonso, Marcelo y Finn, Edward, Física (volumen 1-mecánica-), Editorial Addison Wesley Iberoanericana, 1986.

- Tipler, Paul, Física (volumen 1), Editorial Reverte, 1985.

Noveno grado

- Breijo, Benigno y Zabala, Alfredo, Fisica 9, Editorial Monfort.

- Brett, C. Ely y Suárez William, A., Física 9 Grado-Escuela Básica, 1“ edición, Ediciones Eneva, 1987, Caracas-Venezuela.

- Camero, D. Facundo y Crespo, L. Arturo, Física 9 Grado-Escuela Básica, Caracas-Venezuela.

- Garća, G. Hipólito, Física Básica 9 Grado, Ediciones Eneva, Caracas-Venezuela.

- Mendiola, Esteban, Física. 9 Grado de Educación Básica, lª edición, Editorjal Biosfera, Caracas-Venezuela.

- Requena, O. José M., Fisica. Teorfa. 9 Grado Educación Básica, Editorial Romor.

- Rodríguez, G. Jesús y Reyes Manuel, Fisica. 9 Grado. Tercera Etapa-Educación Básica, Ediciones CO-BO, 1989, Caracas-Venezuela.

- Sánchez Enoc, Física 9 grado, Ediciones CO-BO, Serie Ohm, Caracas-Venezuela, 1988.

\section{Séptimo grado}

- Cáballero, P. Andóśs y Ramos, P. Froilán, A., Estudios de la naturaieza (teoría y práctica). $7^{\circ}$ grado, Editorial Logos, $2^{\mathrm{s}}$ edición.

- Cenicero, Iusto y Álvarez René, Estudio de la naturaleza. 7 grado. Escuela Básica, Editorial Larense, C.A., 1987.

- Feliu Zomaira de, Tíneo Amelia y González Reinaldo, Estudio de la naturaleza. $7^{\circ}$ grado, Ediciones Co-Bo, 1989.

- Fernández Fernando, Estudio de La noturateza. $7^{\circ}$ grado, Ediciones Eneva y Editorial Logos, 1988.

- Guenni Vitali, Proverbio Fulgencio y Martín Reinaldo, Estudio de la naturaleza $7^{\circ}$. Educación Básica, Editorjal Teduca y Santillana, 1989.

- Higuera de Ch. Mery, Sarabia José, Mora de S. Josefa, Estudio de la naturaleza, $7^{\circ}$ grado. Educación Básica, Editorjal Básica (Edibas).

- Hoyos Jesús, F. y Camacaro, Juan, Estudio de la naturaleza. 7 grado, Editorial Eneva, 1991.

- Mazparrote, Serafin y Millán, Justo, Estudio de la naturaleza. $7^{\circ}$ Grado de Educación Básica, Editorial Biosfera.

- Ruiz Álvaro, Estudio de la naturaleza. $7^{\circ}$ Grado, Editorial Triángulo, Caracas-Venezuela, 1988.

de cambio de estado, se transforma y se transmite, depende del sistema de referencia y fijado éste se conserva.»

«Calor es la transferencia de energía de un cuerpo (multiparticulado) a otro determinada exclusivamente por una diferencia de temperatura entre elios.»

De acuerdo con esta definición, fundamentada en la primera ley de la termodinámica $(\Delta \mathrm{E}=\mathrm{W}+\mathrm{Q})$, el calor no es una forma de energía sino que, al igual que el trabajo, modifica la energía de un sistema mediante una transfe- rencia de la misma. Así, los términos de energía calórica, energía no almacenada, energía en tránsito, y términos similares, para referirse al calor, son inadecuados.

\section{RESULTADOS Y DISCUSIÓN}

La presentación de resultados se hará por bloque de concepto, sin un señalamiento específico a cada autor (salvo en aquellos casos donde sea necesario para el 
Tabla II

Relaciones entre subleguaje paradigmático y no paradignático.

\begin{tabular}{|c|c|c|c|c|}
\hline Término & $\begin{array}{l}\text { Símbolo, } \\
\text { especificaciones } \\
\text { equivalente }\end{array}$ & $\begin{array}{l}\text { Sublenguaje no } \\
\text { paradigmático }\end{array}$ & $\begin{array}{l}\text { Sublenguaje } \\
\text { paradigmático }\end{array}$ & Justificación teórica \\
\hline Energía & $\mathrm{E}$ & $\begin{array}{l}\text { Energía es la capacidad } \\
\text { para hacer trabajo }\end{array}$ & $\begin{array}{l}\text { Definición paradigmática } \\
\text { señalada con anterioridad } \\
\text { en el trabajo }\end{array}$ & $\Delta E=W+Q$ \\
\hline Calor & $Q$ & $\begin{array}{r}\text { Flujo de energía } \\
\qquad \Delta \mathrm{E} / \Delta \mathrm{t}\end{array}$ & $\begin{array}{l}\text { Proceso de transferencia } \\
\text { de energía debido a una } \\
\text { diferencia de temperatura }\end{array}$ & $\Delta \mathrm{E}=\mathrm{W}+\mathrm{Q}$ \\
\hline Flujo de calor & $\begin{array}{c}\text { Comiente calorifica } \\
\qquad \mathrm{Q} / \Delta \mathrm{t}\end{array}$ & $\begin{array}{l}\text { Flujo de calor o tasa de } \\
\text { transferencia de calor }\end{array}$ & $\begin{array}{l}\text { No debe hablarse de flujo } \\
\text { de calor, sino de tasa de } \\
\text { transferencia de energía } \\
\text { debido a la diferencia de } \\
\text { temperatura }\end{array}$ & $\Delta \mathrm{E} / \Delta \mathrm{t}=\mathrm{W} / \Delta \mathrm{t}+\mathrm{Q} / \Delta \mathrm{t}$ \\
\hline $\begin{array}{l}\text { Mecanismo de } \\
\text { propagación del calor }\end{array}$ & $\begin{array}{l}\text { Conducción } \\
\text { Convención } \\
\text { Radiación }\end{array}$ & $\begin{array}{l}\text { Mecanismos de } \\
\text { propagación del calor }\end{array}$ & $\begin{array}{l}\text { Mecanismos de } \\
\text { transferencia de la energía } \\
\text { térmica }\end{array}$ & $\Delta \mathrm{E} / \Delta \mathrm{t}=\mathrm{W} / \Delta \mathrm{t}+\mathrm{Q} / \Delta \mathrm{t}$ \\
\hline $\begin{array}{l}\text { Calor absorbido } \\
\text { Calor cedido } \\
\text { Calor suministrado } \\
\text { Calor sustraído }\end{array}$ & $\begin{array}{l}Q_{A} \\
Q_{C} \\
Q_{S M} \\
Q_{S T}\end{array}$ & $\begin{array}{l}\text { El calor puede estar } \\
\text { contenido en los cuerpos }\end{array}$ & $\begin{array}{l}\text { El calor no está contenido } \\
\text { en los cuerpos, porque no } \\
\text { es una función de estado. } \\
\text { Se debe hablar de aumento } \\
\text { o disminución de la } \\
\text { energía }\end{array}$ & $\begin{array}{l}\text { El calor depende del } \\
\text { proceso que siga un } \\
\text { sistema para ir de un } \\
\text { estado a otro }\end{array}$ \\
\hline Energía calórica & $Q$ & $\begin{array}{l}\text { El calor es una forma de } \\
\text { energfa }\end{array}$ & $\begin{array}{l}\text { El calor no es energía, es } \\
\text { un mecanismo para } \\
\text { transferir la energía }\end{array}$ & $\Delta \mathrm{E}=\mathrm{W}+\mathrm{Q}$ \\
\hline Energía térmica & Calor o energía calorica & Calor & $\begin{array}{l}\text { La energía térmica es } \\
\text { aquella parte de la } \\
\text { energía intema de un } \\
\text { sistema que depende } \\
\text { exciusivamente de la } \\
\text { temperatura }\end{array}$ & $\begin{array}{l}\text { Por ejemplo, en un gas } \\
\text { ideal: } \\
\qquad \overrightarrow{\mathrm{E}}_{\text {térmica }}=3 \mathrm{~K} T / 2\end{array}$ \\
\hline Energía interna & Energía térmica & $\begin{array}{l}\text { La energía interna es } \\
\text { siempre de carácter } \\
\text { témico }\end{array}$ & $\begin{array}{l}\text { La energía interna sólo } \\
\text { algunas veces es } \\
\text { exclusivamente de } \\
\text { carácter térmuco }\end{array}$ & $E_{\text {interta }}=\Sigma E c+E p_{\text {config }}$ \\
\hline Calentar & Calor & Proporcionar calor & $\begin{array}{l}\text { Aumentar } \\
\text { la temperatura }\end{array}$ & $\begin{array}{l}\text { El calor no puede ser } \\
\text { proporcionado porque no } \\
\text { puede ser poseido }\end{array}$ \\
\hline
\end{tabular}

entendimiento del contenido de este trabajo), ya que no nos preocupa en esta investigación cuantificar ni identificar la posición de los autores respecto a un determinado concepto, sino señalar las diversas conceptualizaciones que existan. Un análisis detallado de cada libro de la escuela básica se realizó en otro trabajo (Michinel y D'Alessandro 1993).

\section{Del concepto de energía}

En los libros de nivel universitario y en los de los años superiores de la escuela básica analizados no se define de manera explícita la energía a pesar de que el têrmino es profusamente utilizado, ya que se hace referencia a vocablos tales como: energía, energía cinética, de movi- 
Tabla III

Características del concepto de energía, trabajo y calor expresadas por los libros-textos.

\section{Energía}

- Es la capacidad para hacet trabajo.

- Es una magnitud física que se presenta en diversas formas.

- Involucrada en los procesos de cambio de estado (fundamentalmente mecánicos y en algunos textos se consideca que deben ser exclusivamente mecánicos).

- Puede ser o no una función de estado.

- Se transforma.

- Se transmite.

- Se conserva.

\section{Trabajo}

- Es un proceso para transformar, transmitir o intercambiar la energía.

- Es una forma de energía.

- Su magnitud se expresa en las mismas unidades que la energía.

- Involucrado en fenómenos fundamentalmente mecánicos y en algunos libros de textos se señala que exclusivamente mecánicos.

- Algunos expresan la idea de su posesión por los cuetpos.

\section{Calor}

- Es un proceso para tranșferir la energía.

- Es una forma de energía.

- Es poseído por los cuerpos.

- Se presenta cuando existe una diferencia de temperatura entre sistemas.

- Su magnitud se expresa en las mismas unidades que la energía.

miento, potencial, térmica, calórica, en tránsito, interna, eléctrica, química, mecánica, luminosa, sonora, estađos energéticos, etc. El hecho de que el término pueda ser definido en niveles anteriores no es una justificación suficientemente razonable, ya que otros términos igualmente definidos en esos niveles (por ejemplo, fuerza y calor) son nuevamente definidos en los textos de este nivel. Además los problemas que se presentan con el concepto de energía en los niveles previos son suficientemente graves (y semejantes a los que señalamos más adelante) para que sea necesario abordarlos, nuevamente, de forma adecuada.

En los libros correspondientes a los cursos más bajos de la escuela básica se conceptualiza la energía como la capacidad para realizar trabajo, idea que ha sido clara-
Tabla IV

Características de los conceptos de energía, trabajo y caior, a la luz de paradigmas vigentes.
Energía
- Es una magnitud física que se presenta en diversas formas.
- Involucrada en los procesos de cambio de estado (mecánjeos o no).
- Es una función de estado
- Se transforma.
- Se transmite.
- Depende del sistema de referencia.
- Se conserva (en un sistema de referencia fijo).
- Está asociada a tun «sijeto» (onda o partícula) para su transmi- sión o intercambio.
Trabajo
- Es un proceso para transformar, transmitir o intercambiar la energía.
- No es una función de estado.
- No es una forma de energía.
- No se conserva.
- Su magnitud se expresa en las mismas unidades que la energía.
- Involucrado en fenómenos mecánicos o no.
- No lo poseen los cuerpos.
Calor
- Es un proceso para transferir la energía.
- Se presenta s6́lo cuando existe una diferencia de temperatura entre sistemas multiparticulados interactuantes.
- No es una función de estado.
- No es una forma de energía.
- No se conserva.
- Su magnitud se expresa en las mismas unidades que la energía.
- Involucrado en fenómenos mecánicos o no.
- No lo poseen los cuerpos.

mente analizada y rebatida por distintos autores (Lehrman 1973, Hierrezuelo y Montero 1988), debido a su contradicción interna, insuficiencia conceptual e inconsistencia física. Las tablas III y IV, que resumen las características de los conceptos de energía, calor y trabajo, asignadas en los textos analizados y vista a la luz de los paradigmas vigentes, muestran lo señalado.

En algunos de los textos de nivel universitario se señala en forma dispersa algunas de las propiedades que carac- 
terizan la energía (la energía se presenta en varias formas, se conserva, se transforma y se transmite), pero no se expresa, como lo señalamos antes, un concepto completo de ella.

En algunos casos se expresa la idea utilitaria del término cuando se relaciona, parafraseándose su acepción cotidiana, con el costo de los combustibles y su uso de distintas maneras (electricidad en el hogar, alimentos, etc.). Esto se relaciona con las concepciones previas diagnosticadas en estudiantes (Hierrezuelo y Montero 1988), en las cuales se considera la energía como una clase muy general de combustible, frecuentemente relacionado con aquellos procesos que hacen confortable la vida. Se considera la energía como el combustible en sí mismo y no como una propiedad de él. Igualmente coincide con la identificación que hacen los estudiantes, de la energía, con algo necesario para que las cosas trabajen, sobre todo las cosas que producen «confort». De allí que consideren que los automóviles, aviones... tienen energía; pero un objeto en reposo, las nubes, por ejemplo, no hacen trabajo para nosotros $y$, por lo tanto, no tienen energía.

Se llega a señaiar que el concepto de energía es consecuencia de otros conceptos (fuerza, movimiento) o leyes, manteniendo la idea histórica de su aparición a partir del concepto de vis viva (Holton 1986). Coincide esta idea, detectada en los textos analizados, con la identificación entre fuerza y energía encontrada en los estudiantes (Duit 1984).

\section{Del trabajo}

En la totalidad de los textos analizados se considera el trabajo como una forma de energía. En algunos se presenta la idea de manera bastante contradictoria, ya que, si en un principio tratan de zafarse de esta concepción, durante el desarrollo del tema regresan a ella, consiguiéndose párrafos como el siguiente:

«De manera análoga, el trabajo no es algo que el sistema contenga en una cantidad definida. Sobre un sistema se puede efectuar una cantidad definida de trabajo, tal como lo ilustra el apartato de Joule. El trabajo, como el calor, involucra una transferencia de energía. En la mecánica, el trabajo interviene en aquellas transferencias de energía en la que no intervenga la temperatura. Si se transmite energía calorífica por diferencia de temperatura, el calor puede distinguirse del trabajo definiendo el trabajo como la energía que se transmite de un sistema a otro de tal manera que no esté involucrada directamente una diferencia de temperatura...» (Resnick-Halliday).

Son evidentes las contradicciones internas en el párrafo, ya que no sólo los sistemas no contienen una cantidad definida de trabajo, sino que no tienen trabajo; los sistemas tienen energía y ellos (o sobre ellos) hacen (o se puede hacer) trabajo para intercambiar esta energía con otros sistemas.

Mediante la siguiente cita podemos ejemplificar lo que se ha señalado:
«Se ha visto que es posible transferir energía entre un sistema y su entorno, de dos maneras: una es trabajo realizado por (o sobre) el sistema; este modo de intercambio conduce a cambios mensurables en las variables macroscópicas del sistema, como la presión y el volumen del gas; la otra es la transferencia de calor, la cual se lleva a cabo a nivel microscópico y se manifiesta por cambios en la temperatura...» (Serway).

\section{Sin embargo el autor ha señalado antes que:}

«... se ve que los efectos de realizar trabajo mecánico sobre un sistema y los de agregar calor directamente, como una llama, son equivalentes. Es decir, tanto el calor como el trabajo son formas de energía...»

Se evidencia una permanente confusión entre forma de energía y forma de intercambio o transferencia de la energía. La siguiente analogía puede aclararnos aún más el problema: una mercancía puede ser transportada de un sitio a otro por medio de un automóvil, un avión, un animal de carga, etc.; es erróneo afirmar que los medios de transporte (el trabajo o el calor) utilizados son la mercancía (la energía). Así mismo, ni el calor ni el trabajo son energía aunque tengan las mismas unidades que ella.

Otra situación que se presenta es el tramposo artificio que se utiliza para analizar los efectos (el trabajo) de la llamada fuerza de roce para que emerja el término de calor. En la mayoría de los textos analizados se desarrolia el tema del trabajo aplicado a una partícula (puntual) y de repente, sin que medie mayor explicación, se habla de una fuerza de roce (que es un promedio de las fuerzas intermoleculares que operan ctando dos superficies están en contacto) sobre una partícula, que produce una «disipación de energía» y un aumento de energía interna, evento característico de cuerpos masivos (multipartículas) y no de una partícula. Si uno de los cuerpos es una partícula, no se ejercerá sobre ella ninguna fuerza de roce, debido a que no posee superficie. Quizás la falsa concepción provenga del hecho de que la fuerza de roce deslizante no dependa de la superficie de contacto entre los cuerpos, lo cual es obviamente cierto, ya que, como señalamos antes, es un promedio de superficie. Pero, si no hay superficie de contacto (porque uno de los cuerpos es una partícula), no existen fuerzas intermoleculares y tampoco fuerza de roce.

En la mayoría de los textos analizados se hace un uso limitado del concepto de trabajo cuando se refiere exclusivamente al trabajo externo. De modo que se excluyen trabajos del tipo muscular, químico, etc. Pareciera que los sistemas activos, como por ejemplo el músculo, no realizan trabajo.

\section{Del calor}

En el concepto de calor se profundizan las confusiones que se han señalado respecto al trabajo. Se le define, de manera incorrecta, como una forma de energía, en un momento, y, en otro, se le considera como un mecanismo. Por ejemplo, dos textos señalan: 


\section{INVESTIGACIÓN Y EXPERIENCIAS DIDÁCTICAS}

«Como vimos en el capítulo 16, se suministra o se quita calor a un sistema en virtud de la diferencia de temperatura que existe entre él y su medio exterior. El calor se considera como positivo cuando entra al sistema, y como negativo cuando sale. Desde el punto de vista de la termodinámica, trabajo y calor son medios de transferir energía; esto es, métodos mediante los cuales puede aumentarse o disminuirse la energía de un sistema.» (Sears-Zemansky).

"Actualmente admitimos que entre dos cuerpos que se encuentran en estas condiciones existe un flujo de energía, a la cual llamamos calor.» (Sears-Zemansky).

«El calor es la energía transferida entre dos sistemas y que está exclusivamente relacionada con la diferencia de temperatura existente entre eilos.» (Sears-Zemansky).

«La teoría del calórico sobre el calor continuó siendo prestigiosa incluso cuarenta años después de realizado el trabajo de Thompson, pero fue gradualmente cayendo en desuso a medida que eran observados más ejemplos de la no conservación del calor. Hasta 1840 no floreció la teoría mecánica moderna. Desde su punto de vista, el calor es otra forma de energía, intercambiable con las diversas formas de energía mecánica ya discutidas.» (Tipler).

«Los experimentos de Joule y otros experimentos demostraron que ni el calor ni la energía mecánica se conservan independientemente, pero que la energía mecánica perđida iguala el calor producido (medidos en las mismas unidades). Lo que se conserva es el total de la energía mecánica y la energía térmica.» (Tipler).

«Si añadimos calor al agua, elevamos su temperatura, pero también podemos elevar su temperatura agitando o realizando trabajo sobre el agua de alguna forma que no se suministre calor.» (Tipler).

El calor es interpretado, y así se expresa en la totalidad de los textos analizados, como una propiedad de los cuerpos y no como un mecanismo para la transferencia de energía térmica. Expresiones tales como: depósito de calor, cantidad de calor de un cuerpo, calor que fluye, calor que se transfiere, calor que se pierde o se gana, calor generado por un cuerpo, calor que se agrega o se suprime, calor que se extrae, calor que se conduce, calor que entra o sale de un cuerpo, calor que se intercambia, que se absorbe, que se desprende, calor devuelto, calor que se propaga... son muy comunes y reflejan la falsa idea de que el calor es una propiedad de los cuerpos. Si fuera así, es evidente que se pudiera medir el calor de un cuerpo o de un sistema en un momento dado de alguna manera, aunque fuera teóricamente.

Se confunde en muchos casos el calor con la energía térmica y hasta se le asigna el término de energía calorífica o calórica.

Se considera el calórico como un paradigma superado, y en algunos de los textos analizados se señala explícitamente, pero se continúa utilizando algunos de los términos que son característicos de esta teoría; por ejemplo: capacidad calórica, flujo de calor, calor latente, calor escondido, cantidad de calor, calor específico, calor de fusión, calor de vaporización, calor de condensación, calor de solidificación, flujo calorífico, corriente calorífica.

En la gran mayoría de los libros analizados se habla de procesos de transmisión, propagación o mecanismos de transmisión del calor para referirse a la convección, conducción y radiación. Estos señalamientos son incorrectos porque el calor es un proceso y no una energía; más adecuado sería hablar de procesos de intercambio térmico o procesos para la variación de la temperatura.

Algunos libros analizados de la escuela básica, para ilustrar el concepto de calor y con el fin de diferenciarlo de la temperatura, hacen uso de analogías tales como: calor es a cantidad de líquido como temperatura es a nivel del líquido, promoviendo la falsa idea de que los cuerpos poseen calor como el líquido posee masa.

Se evidencia, a través de los resultados, la existencia de interpretaciones o concepciones previas, que para entenderlas de manera sistemática pueden ser clasificadas en tres tipos:

- Un primer tipo que expresa deficiencias del autor en el conocimiento de las teorías físicas vigentes. Un ejemplo es cuando el autor expresa la posibilidad de medir el calor y lo confunde con la temperatura; se evidencia la creencia de que existe una sustancia material, como el calórico, que es poseída por los cuerpos en mayor cantidad cuando está a mayor temperatura y viceversa.

- Un segundo tipo tiene características de las interpretaciones o concepciones previas típicas. Así pudieran ser consideradas las posiciones de autores que reconocen, por ejemplo, que la teoría cinética es el paradigma científico para interpretar los procesos de intercambio de energía térmica y, sin embargo, utilizan términos que rememoran la teoría del calórico.

Coincidimos con otros autores (Hierrezuelo y Montero 1988) cuando señalan: «Un error frecuente entre los alumnos, y que también se encuentra en algunos libros de textos, es confundir energía con trabajo. Pensamos que en esto influye la definición usualmente utilizada en los libros: energía es la capacidad para realizar trabajo. Se consideran el calor y el trabajo como otras formas de energía. Esta interpretación es incorrecta, ya que sabemos que tanto el calor como el trabajo son nombres que damos a dos tipos de procesos de intercambio de energía. No podemos decir que la energía se transforma en trabajo. La energía puede transformarse o transmitirse por medio del trabajo, pero nunca transformarse en trabajo. El trabajo sea mécánico, eléctrico o magnético se refiere siempre a un proceso.»

- Un tercer tipo refleja la existencia de un sublenguaje, ya tradicional pero deficiente, donde influye, en forma determinante, por un lado la permanencia de la concepción mecanicista de la energía y por el otro la teoría del calórico. La persistencia de tal sublenguaje, creemos 
que se debe a que abrevia o simplifica, en algunos casos, la explicación de ciertos fenómenos físicos. Tal abreviación o simplificación, aunque puede tener utilidad didáctica, refuerza las falsas concepciones.

Este tipo de interpretación se hace evidente cuando se hace un análisis historico de la aparición de los conceptos de energía, trabajo y calor, y se constata que desde el propio origen de tales términos hay una influencia que frena el desarrollo de los mismos e impide una coherencia con los paradigmas actuales. El concepto de energía nace limitado por la concepción mecanicista, derivado, como ya lo señalamos, del concepto de fuerza viva o vis viva. El término trabajo se origina limitado al trabajo externo, el de calor a las concepciones que se tenían antes de la intepretación de los experimentos de Thompson y ambas están condenadas a nuestro modo de ver por una interpretación incorrecta de la primera ley de la termodinámica ( $\Delta \mathrm{E}=\mathrm{W}+\mathrm{Q})$. Dicha interpretación considera el trabajo W y el calor Q como formas de energía y no como los posibles procesos para que se produzca la variación de energía $\Delta \mathrm{E}$; la ecuación señalada no indica que $W+Q$ es la energía sino que $W$ y $Q$ generan un cambio en la energía.

Este último tipo de interpretación previa es tal vez el más importante porque es un factor de generación de los otros dos. Un ejemplo de ello es la resistencia al cambio del generalizado concepto de que «la energía es la capacidad para hacer trabajo", a pesar de las profundas críticas que se le han hecho. De allí que propongamos la necesidad de construir un nuevo sublenguaje que permita la sustitución del actual.

La recurrencia en lo que los autores opinan de la energía, trabajo, calor y otros conceptos afines nos llevó a la necesidad de hacer una revisión histórica de la representación que pudieran tener los creadores o promotores, más relevantes, de estos conceptos. Esa revisión es resumida a continuación.

\section{VIS VIVA Y LA ENERGÍA CINÉTICA}

El principio de conservación de la energía tiene sus orígenes en el planteamiento (1669) de Huygens (16291695 ), el cual propuso que en el caso especial de choques perfectamente elásticos, además de la cantidad de movimiento, se debía conservar otra magnitud: «la vis viva o fuerza viva», el producto de la masa por la velocidad al cuadrado. Posteriormente, se introduce el concepto de trabajo y se obtuvo el resultado:

$W=$ Trabajo $=$ Fuerza. Desplazamiento $=1 / 2(\Delta v i s$ viva)

que no es otra cosa que el teorema del trabajo y la energía cinética aplicada a una partícula:

$W_{\text {exierna }}=\Delta E c_{\text {paricuta }}$

\section{LA TEORÍA DEL CALÓRICO}

Según Holton (1986) «los antiguos atomistas griegos explicaron las diferencias de temperatura de los cuerpos - Ia denominada intensidad o grado de calor- por un esquema conceptual, imaginando el calor como una sustancia especial, no perceptible directamente, atómica en estructura como las restantes, que se difundía a través de los cuerpos rápidamente y que, posiblemente poseía algún peso». Posteriormente, en el siglo Xvil, se tenía una idea más precisa (Holton 1986), «se trataba de un fluido, tenue, capaz de entrar y salir a través de los "poros" más pequeños, y cuya magnitud dependia de la temperatura, era imponderable y, quizás, semejante al fluido sutil y omnipresente de Descartes o los éteres invocados por alguien para explicar la gravitación, Ia propagación de la luz y el calor radiante, la transmisión de las fuerzas eléctricas y magnéticas, etc.».

La consolidación de la teoría del calórico, especialmente debido a los resultados concluyentes que se obtuvieron al colocar en contacto cuerpos a diferentes temperaturas (Black 1728-1799) concluyeron en la ley de la conservación del calor (o del calórico): «El calor ni se crea ni se destruye, pero sí puede ser transferido de un cuerpo a otro.»

En términos formales:

$\Delta Q_{A}+\Delta Q_{B}=0$

con

$\Delta Q(\mathrm{cal})=\mathrm{M}_{\text {muestra }}(\mathrm{g}) \times \mathrm{Ce}(\mathrm{cal} / \mathrm{g} . \mathrm{C}) \times\left[\mathrm{T}_{\text {final }}\left({ }^{\circ} \mathrm{C}\right)-\mathrm{T}_{\text {inicial }}\left({ }^{\circ} \mathrm{C}\right)\right]$,

donde A y B son dos cuerpos, por ejemplo, un bloque caliente de metal y una cierta cantidad de agua fría. La ecuación anterior significa que el calor perdido por A es igual al calor ganado por $B$, y se cumple siempre y cuando el sistema formado por los dos cuerpos esté aislado y no haya cambios químicos ni de estado físico. Obsérvese que el hecho de suponer que $\mathrm{Q}$ es proporcional a $T$ sugiere, como de hecho to asumieron erróneamente los seguidores de la teoría del calorico, que el calor es poseído por los cuerpos ( $Q$ es proporcional a $T$ ). Formalmente, si escribimos $\Delta Q=M_{m} \mathrm{Ce}\left(\mathrm{T}_{\mathrm{f}}-\mathrm{T}_{\mathrm{i}}\right)$, entonces, se puede asumir falsamente $Q_{\mathrm{f}}=\mathrm{M}_{\mathrm{m}} \mathrm{CeT}_{\mathrm{f}}$ y $\mathrm{Q}_{\mathrm{i}}=$ $\mathrm{MCeT}_{i}$ como una propiedad del cuerpo.

Black introdujo los términos caloría, capacidad calorífica, calor latente (o calor de fusión) y calor sensible. La conservación del calórico fue sometida a pruebas experimentales, entre otros científicos, por Benjamín Thompson, conde de Rumford (1753-1814).

\section{LOS EXPERIMENTOS DE RUMFORD}

Estos experimentos (indagación concerniente al origen del calor generado por fricción [1796] y el artículo publicado por el London Philosophical Transaction [1799] se pueden clasificar en dos grupos. Uno de ellos demostró la imponderabilidad del calórico y el otro su no 
conservación. En uno de los experimentos de este último grupo (realizado por Thompson, en Munich, cuando fue comisionado por el gobierno de Baviera para dirigir una fábrica de cañones), con el fin de evitar el calentamiento excesivo, el taladro del cañón se mantenía lleno de agua, y como ésta hervía durante el proceso de taladrado, el depósito tenía que llenarse continuamente. Se admitía que para hervir el agua había que suministrarle calórico y la producción continua del mismo se explicaba por la hipótesis de que cuando la materia se divide finamente (como sucedía en el proceso de taladrado) disminuye su capacidad para retener el calórico, el cual, desprendido đe esta forma, hacía hervir el agua. Rumford observó, sin embargo, que el agua de refrigeración continuaba hirviendo cuando la herramienta se ponía tan roma (desgastada) que no cortaba. Esto es, la herramienta roma constituía, todavía, aparentemente un depósito inextinguible de calórico mientras se realizara trabajo mecánico para hacer girar la herramienta. Con este experimento se demostró que el «calórico» podía ser creado por fricción y no había una desaparición correspondiente de calórico en ningún otro sitio (y, por lo tanto, no existía conservación). Rumford llegó con este y otros experimentos a la misma conclusión de Galileo y Newton, que sostenían (sin comprobación experimental) que el calor era una manifestación del movimiento molecular atómico.

\section{EL CALOR RADIANTE}

Debido a que se pensaba que el calor viajaba a través del espacio (que se suponía que era la región comprendida entre el Sol y la Tierra), el calor no pođía ser simplemente, un modo de movimiento de la materia, sino una sustancia diferente. De allí pudiera derivarse la falsa concepción de la radiación como una forma de propagación del calor, que es usual encontrar en los libros de texto de física de diferentes niveles. Como los experimentos realizados durante las primeras tres décadas del siglo xIx demostraron que el hoy mal denominado «calor radiante» poseía todas las características de la luz (reflexión, refracción, interferencia, polarización, etc.), se produjo por extension natural, para ese entonces, que cualquier tipo de calor tenía las propiedades de la luz. Actualmente sabemos que el tanto la luz (visible) y el «calor radiante» son ambas radiación electromagnética. Como la teoría corpuscular (de Newton) sobre la luz era dominante, se sostuvo que el calor era también una sustancia compuesta de partículas. Una vez que la teoría corpuscular fue desplazada por la teoría ondulatoria, se sostuvo (después de 1825) una teoría ondulatoria del calor (en la cual era considerado como un movimiento vibratorio del éter).

\section{VON MAYER. JOULE. EL EQUIVALENTE MECANICODEL CALOR. VONHELMHOLTZ}

\section{Mayer (1814-1878)}

Mayer sostuvo junto con Rumford que el calor debía ser una forma de movimiento y obtuvo, utilizando el expe- rimento de expansión libre đe Gay Lussac (Holton 1986), una equivalencia entre el calor y el trabajo de: 1 caloría $=3,6$ joules. Mayer extendió su principio de indestructibilidad y convertibilidad de la energía a los procesos químicos, astronómicos y biológicos.

\section{James Prescot Joule (1818-1889)}

Joule obtuvo, a través de una serie de experimentos, el denominado equivalente mecánico del calor. «Cuando el trabajo mecánico se convierte en calor, en cualquier circunstancia, la razón entre el trabajo realizado y el calor desarrollado tiene un valor constante y mensurable.» El valor aceptado hoy día es 4,184 joule/cal. Uno de los experimentos más importantes efectuados por Joule ha sido descrito por George Gamow en su libro Biografía de la Física: «Para sus experimentos, Joule empleaba un aparato que consistía en una vasija Ilena de agua que contenía un eje giratorio con varias paletas batidoras insertas en él. El agua de la vasija no podía girar libremente al mismo tiempo que las paletas por virtud de unas tablillas especiales insertas en las paredes de las vasijas que aumentaban la fricción interior. El eje con sus paletas era movido por un peso suspendido a través de una polea, y el trabajo efectuado por el peso en su descenso era transformado en calor de rozamiento que se comunicaba al agua. Conociendo la cantidad de agua en la vasija y miđiendo la elevación de la temperatura, Joule podía calcular la cantidad total de calor producido. Por otra parte, el producto del peso impulsor por la distancia de su descenso suministraba el valor del trabajo mecánico.»

Es importante observar el uso inapropiado del término «equivalente mecánico del calor», ya que en el experimento en cuestión el trabajo no se «convierte» en calor sino que produce una variación đe la energía interna. Es un experimento donde no existe un proceso (calor) de cambio de energía como producto de una diferencia de temperatura.

\section{Herman Von HeImholtz (1821-1894)}

Este científico alemán demostró la validez del principio de conservación de la energía y extendió la validez del principio a los diversos campos de la física. En un artículo publicado en 1861 en Proc. of the Royal Institution lo aplicó a la fisiología.

Como emerge del resumen anterior, el análisis histórico pone de manifiesto que desde el propio origen de los conceptos que son objeto de estudio en este trabajo se ha desarroilado un sublenguaje inadecuado. Así que, parece necesario promover la formulación de un nuevo sublenguaje que sea coherente con los paradigmas actuales de la física y que no sea fuente de preconcepciones acerca de la energía y otros conceptos afines.

La tabla II resume el inicio de creación de ese subienguaje alternativo, claro y coherente con los paradigmas 
científicos actuales (la ley general de la cconservación de la energía y la teoría cinética molecular).

Igualmente, el desarrollo matemático que se formula abajo, y que permite establecer una relación adecuada entre la conservación de la energía y la primera ley de la termodinámica, es también parte del sublenguaje que debe desarrollarse, ya que las deficiencias del viejo sublenguaje no sólo están en el marco del lenguaje gramatical, sino también en los aspectos algrebraicos del mismo.

\section{LA CONSERVACIÓN DE LA ENERGÍA Y LA PRIMERA LEY DE LA TERMODINÁMICA}

Actualmente sabemos que la primera ley de la termodinámica es la extensión, a un sistema de muchas partícu* las, del teorema del trabajo y de la energía cinética, con el añadido de una fuente termica. En lo que sigue deduciremos dicha ley, comenzando con el teorema del trabajo y la energía cinética. Si el sistema considerado es de muchas partículas.

$\mathrm{W}_{\text {total }}=\mathrm{W}_{\text {externo }}+\mathrm{W}_{\text {interno }}=\Delta \mathrm{Ec}_{\text {sistema }}$

1. En el caso especial de una partícula:

$\mathrm{W}_{\text {total }}=\mathrm{W}_{\text {extemo }}=\Delta \mathrm{Ec} c_{\text {particula }}$

ya que no puede existir trabajo interno porque no existen fuerzas internas. En este caso, también se obtiene:

$\mathrm{W}_{\text {no conservativo }}=\Delta \mathrm{Em}_{\text {particula }}$

donde hemos considerado que:

$W_{\text {total }}=W_{\text {conservativo }}+W_{\text {no conservativo }}$

y

$\mathrm{W}_{\text {conservativo }}=-\Delta \mathrm{Ep}$,

y la energía mecánica de la partícula es

$\mathrm{Em}=\mathrm{Ep}+\mathrm{Ec}=$ Energía potencial + Energía cinética

2. Si tenemos muchas partículas:

$\mathrm{W}_{\text {interno }}=-\Delta \mathrm{Ep}_{\text {inienta }}$

donde hemos supuesto que todas las fuerzas internas son conservativas. Así que el teorema del trabajo y la energía cinética adopta la siguiente forma:

$\mathrm{W}_{\text {extemio }}=-\Delta \mathrm{Ec}_{\text {sistema }}+\Delta \mathrm{Ep}_{\text {interna, sistema }}$

si definimos:

$\mathrm{W}_{\text {propia sistema }}=\mathrm{Ec}_{\text {sistema }}+\mathrm{Ep}_{\text {interna, sistema }}$

entonces:
$\mathrm{W}_{\text {extemo }}=\Delta \mathrm{E}_{\text {propis sistema }}$

con

$E_{\text {propia, siscema }}=\sum_{i} \frac{1}{2} m_{i} v_{i}^{2}+\sum_{i j} E p_{i j}$, la segunda sumatoria es efectuada $\sin$ repetir pares.

Si bajo cualquier circunstancia,

$\mathrm{W}_{\text {externo }}=0$

entonces, la energía propia del sistema es constante. Este resultado también se obtiene cuando el sistema está aisłado.

3. Si tenemos muchas partículas y además existe una fuente térmica, se cumple que:

$\mathrm{W}_{\text {cxterno }}+\mathrm{Q}=\Delta \mathrm{E}_{\text {propia sistema }}$

Considerando que la energía cinética del sistema se puede descomponer como:

$$
E c_{\text {sistema }}=E c_{\text {interme }}+E c_{\text {ccnero-mass }}=\sum_{1} \frac{1}{2}-m_{i}\left(v_{i}^{f}\right)^{2}+\frac{1}{2}-M_{\text {cotas }}\left(v_{\text {cm }}\right)^{2}
$$

donde el primer término es la energía cinética de las partículas referidas al centro de masa del sistema y el segundo término es la energía cinética de traslación del centro de masa respecto a un referencial externo. De esta manera podemos reescribir:

$\mathrm{W}_{\text {externo }}+\mathrm{Q}=\Delta \mathrm{E}_{\text {interna sistema }}+\Delta \mathrm{Ec}_{\text {centro de mase }}$

con:

$\mathrm{E}_{\text {interna sistema }}=\mathrm{Ec}_{\text {imterna }}+\mathrm{Ep}_{\text {interna sistera }}$

Si el trabajo externo se descompone en trabajo de las fuerzas conservativas y trabajo de las fuerzas aplicadas (no conservativas y fuerzas de las cuales no sabemos su origen), entonces:

$W_{\text {appiteda as sistema }}+W_{\text {conservativo }}+Q=\Delta E_{\text {interna sistema }}+\Delta E c_{\text {centro de masa }}$ como

$\mathrm{W}_{\text {conservativo }}=-\Delta \mathrm{Ep_{ \text {externa } }}$

entonces:

$\mathrm{W}_{\text {apliczdota al sistema }}+\mathrm{Q}=\Delta \mathrm{E}_{\text {interna sistema }}+\Delta \mathrm{Ec}_{\text {centro de masa }}+\Delta \mathrm{Ep}_{\text {externa }}$

Si definimos:

$\mathrm{E}_{\text {total sistema }}=\mathrm{E}_{\text {interna sistemat }}+\mathrm{Ec}_{\text {centro de masa }}+\mathrm{EP}_{\text {externa }}$

y también:

$\mathrm{E}_{\text {tolal sistemat }}=\mathrm{E}_{\text {propia }}+\mathrm{Ep}_{\text {exierna }}$ 
cont:

$\mathrm{E}_{\text {propia }}=\mathrm{E}_{\text {interna sistema }}+\mathrm{Ec}_{\text {centso de masa }}$

entonces:

$W_{\text {aplicado al sistema }}+Q=\Delta E_{\text {total sistema }}$

que es la formulación general actual de la primera ley de la termodinámica.

\section{CASOS ESPECIALES}

Si todas las fuerzas externas son conservativas, entonces:

$\mathrm{W}_{\text {aplicado }}=0$ y $\Delta \mathrm{E}_{\text {tocal sistemat }}=\mathrm{Q}$

si además $Q=0$, entonces:

$\mathrm{E}_{\text {total sistema }}=$ constante $=\mathrm{E}_{\text {propia }}+\mathrm{Ep}_{\text {externa }}$

Si el sistema está aislado de los alrededores, se cumple que:

$F_{\text {aplicada }}=0, Q=0$ y $W_{\text {aplicado }}=0$

además, se cumple que:

$E p_{\text {extemo }}=0$, ya que $\mathrm{F}_{\text {conservativo }}=0 \mathrm{y} \mathrm{W}_{\text {conservativo }}=0$

Así que:

$\mathrm{E}_{\text {total sistema }}=$ constante $=\mathrm{E}_{\text {propia }}$

Debido a que la fuerza externa es nula

$\mathrm{Ma}_{\mathrm{cm}}=0$, de modo que $\mathrm{a}_{\mathrm{cm}}=0$ ( $\mathrm{a}_{\mathrm{cm}}=$ aceleración del centro de masa)

y

$\mathrm{v}_{\mathrm{cm}}=$ constante $\left(\mathrm{v}_{\mathrm{cm}}=\right.$ velocidad del centro de masa), entonces también se cumple:
$\Delta \mathrm{E}_{\text {iotal sistema }}=\Delta \mathrm{E}_{\text {interna sistema }}$

Así que:

$W_{\text {aplicado }}+Q=0=\Delta E_{\text {tocal sistema }}=\Delta E_{\text {interna sistema }} y$

$\mathrm{E}_{\text {interna sistema }}=$ constante

a) Si Ia fuerza externa neta es nula $\left(F_{\text {externa }}=0\right)$,

entonces:

$\Delta \mathrm{Ec}_{\text {centro de masa }}=0$

y si además se cumple:

b) $W_{\text {conservativo }}=-\Delta \mathrm{Ep}_{\text {externa }}=0$

entonces:

$\Delta \mathrm{E}_{\text {total sistema }}=\Delta \mathrm{E}_{\text {interna sisterna }}+\Delta \mathrm{E}_{\text {centro de masa }}+\Delta \mathrm{Ep}_{\text {exterma }}$

se reduce a:

$\Delta \mathrm{E}_{\text {total sistema }}=\Delta \mathrm{E}_{\text {interna sistema }}$

y así, la primera ley puede escribirse como usualmente se hace en química:

$\mathrm{W}_{\text {aplicada }}+\mathrm{Q}=\Delta \mathrm{E}_{\text {interna sistema }}$

donde $W_{\text {aplicada }}$ y $Q$ son, como lo señalamos antes, procesos que permiten la variación de la energía interna de un sistema multiparticulado. El término $W_{\text {aplicad incluye }}$ todos los tipos de interacción (física, química, fisiológica, etc.) que se pueda expresar como el producto de una fuerza generalizada por un desplazamiento generalizado (trabajo mecánico) (García-Colín 1972, Volkenshtein 1985). Es importante hacer referencia a la reformulación, que se hace en el Alonso-Finn, del principio de Ia conservación de la energía para los sistemas de muchas partículas y que sirvio de referencia para el desarrollo que se hace en este trabajo.

\section{REFERENCIAS BIBLIOGRÁFICAS}

ANDER-EGG, E., 1980. Técnicas de investigación social, 14 edición, (EI Cid Editor: Buenos Aires).

AUSUBEL, D., NOVAK, J. y HANESIAN, H., 1980. Psicología Educacional. (Editorial Interamericana: Río de Janeiro).

BARROW, G.M., 1988. Thermodynamics should be built on energy not on heat and work, $J$. Chem. Educ., 65(2), pp. $122-125$.
BERELSON, B., 1952. Content analysis in communication research. (Free Press: Nueva York).

BOLTZMANN,L., 1986. Escritos de Mecánica y Termodinámica. (Alianza Editorial: Madrid).

DRIVER, R. y WARRINGTON, L., 1985. Student's use of the principle of energy conservation in problems stituations, Physics Education, 20, pp. 171-176. 
DUIT, R., 1984. Learning the energy concept in school, empirical result from Phylippines and West Germany, Physics Education, 19. pp. 59-66.

ERICKSON, G.L., 1979. Children's conceptions of heat and temperature, Science Education, 63(2), pp. 221-230.

GARCÍA COLÍN, S., 1972. Introducción a la Termodinámica Clásica. (Editorial Trillas: Méjico).

GIL PÉREZ, D, 1991. Enseñanza de las Ciencias , en Enseñanza de las ciencias y las matemáticas. Tendencias e innovaciones. Publicado por la organización de Estados tberoamericanos para la educación, la ciencia y la cultura (programa IBERCIMA). (Editorial popular: Madrid).

HIERREZUELO, J. y MONTERO, A., 1988. Energía, en La ciencia de los alumos. Su utilización en la didáctica de la física y la química. (Editorial Laia y Ministerio de Educación y Ciencia: Barcelona).

HOLTON, G., 1986. Introducción a los conceptos y teorías en las ciencias físicas. (Editorial Reverté).

LEHRMAN, R.L., 1982a. Physics texts: An evaluative review, The Physics Teacher, 20, pp. 508-518.

LEHRMAN, R.L., 1982b. Confused physics: A tutorial critique, The Physics Teacher, 20, pp. 519-523.

LEHRMAN, R.L., 1973. Energy is not the ability to do work, The Physics Teacher, 11(15), pp. 15-18.

MICHINEL, J.L., D'ALESSANDRO, A. y ORTEGA, H., 1992. Energía y sistemas: Conceptos relevantes para aprender Física dirigido a estudiantes de Medicina, Revista de Ensino de Física, 14, Brasil.
MICHINEL, J.L., D'ALESSANDRO, A., 1993. Concepciones no formales de la energía en textos de física para la Escuela Básica. Revista de Pedagogia, 33, pp. 41-59. Venezuela.

MOREIRA, M.A., 1982. Energy, entropy and irreversibility. Mimeografiado. (Río Grande del Sur: Brasil).

PIAGET, J., 1969. Psicología y Pedagogía. (Ariel: Barcelona).

QÜENZA, S.E., 1986. La evaluación de los materiales educativos impresos. (Ediciones El Mácaro: Macaray-Venezuela).

SEBASTIÁ, J.M., I982. Bases conceptuales y metodológicas para la evaluación de los libros de texto en el área de las ciencias naturales. Tesis de grado de MSc, UCV.

SEBASTIÁ, J.M., 1987. Algunos patrones de interpretación espontánea frente a siluaciones de dinámica clásica. Mimeografiado, (USB: Caracas-Venezuela).

SOLOMON, J., 1983. Learning about energy: How pupils think in two domains, European Journal of Science Education, 5 , pp. $49+59$.

TOUGER, J.S., 1983. Analyzing the linguistic structure of physical concepts: Possible implications for educational research and for physics pedagogy, International summer workshop: Research on Physics Education. (La Londe les Maures: Francia).

VOLKENSHTEIN, M.V., 1985. Biofísica. (EditoriaI MIR: Moscú).

WATTS, M., 1983. Some alternative views of energy, Physics Education, 18, pp. 213.217.

ZEMANSKY, M.W., 1970. The use and misuse of the word «heat» in physics teaching, The Physics Teacher, pp. 295-300. 